\title{
Hyperthyroidism After Allogeneic Hematopoietic Stem Cell Transplantation: A Report of Four Cases
}

\author{
Erdal Sağ1, Nazlı Gönç2, Ayfer Alikaşifoğlu2, Barış Kuşkonmaz³, Duygu Uçkan33, Alev Özön2, \\ Nurgün Kandemir2 \\ 1 Hacettepe University Faculty of Medicine, Department of Pediatrics, Ankara, Turkey \\ 2 Hacettepe University Faculty of Medicine, Department of Pediatric Endocrinology, Ankara, Turkey \\ 3 Hacettepe University Faculty of Medicine, Department of Pediatric Hematology, Ankara, Turkey
}

\section{WHAT IS ALREADY KNOWN ON THIS TOPIC?}

Thyroid dysfunction is frequently seen after hematopoietic stem cell transplantation (HSCT), however, hyperthyroidism is one of the rarest complications.

\section{WHAT THIS STUDY ADDS?}

Hyperthyroidism developing after HSCT may vary in etiology, severity and need of treatment.

\section{ABSTRACT}

Hematopoietic stem cell transplantation (HSCT) is the only curative treatment for many hematological disorders, primary immunodeficiencies, and metabolic disorders. Thyroid dysfunction is one of the frequently seen complications of HSCT. However, hyperthyroidism due to Graves' disease, autoimmune thyroiditis, and thyrotoxicosis are rare. Herein, we report a series of 4 patients who were euthyroid before HSCT but developed hyperthyroidism (3 of them developed autoimmune thyroid disease) after transplantation.

Key words: Hyperthyroidism, autoimmune, hematopoietic stem cell transplantation, bone marrow transplantation

Conflict of interest: None declared

Received: 03.08.2015

Accepted: 14.08 .2015

\section{Introduction}

Hematopoietic stem cell transplantation (HSCT) remains the only curative treatment for many inherited and acquired pediatric hematological disorders such as haemoglobinopathies, bone marrow failure disorders, primary immunodeficiencies, and metabolic disorders.

Thyroid dysfunction is one of the frequently seen complications of HSCT. Several kinds of thyroid disease such as hypothyroidism, euthyroid sick syndrome, autoimmune thyroiditis, Graves' disease, and thyroid tumors have been reported so far. Hypothyroidism, which is seen in nearly $40 \%$ of patients, is the most common thyroid disease, and it is especially more prevalent among patients receiving total body irradiation $(1,2,3,4,5)$. 
Graves' disease, autoimmune thyroiditis, and thyrotoxicosis are seen rarely, and the underlying mechanisms are either transfer of donor auto-reactive immune cells or immune dysregulation and immune reconstitution secondary to graft-versus-host disease (GVHD) (6). Herein, we report a series of 4 patients who were euthyroid before HSCT but developed hyperthyroidism (3 of them developed autoimmune thyroid disease) after transplantation.

\section{Case Reports}

\section{Case 1}

A ten-month-old female was diagnosed with betathalassemia major and underwent bone marrow transplantation from her HLA-matched mother when she was 29 months of age. Neutrophil and platelet engraftments were observed on the $15^{\text {th }}$ and $33^{\text {rd }}$ days of transplantation, respectively (Table 1 ).

On post-transplant day 25 , she had acute GVHD, presenting with nodular and maculopapular rash, and methylprednisolone was initiated. She did not respond to steroid and cyclosporine A (CsA) treatment. Mycophenolate mofetil was added to the treatment regimen. At $+5^{\text {th }}$ month, she had a seizure with magnetic resonance imaging findings compatible with posterior reversible encephalopathy syndrome (PRES). CsA treatment was replaced by tacrolimus. At $+19^{\text {th }}$ month, a mosaic pattern at thorax high-resolution computed tomography (HRCT) was detected, a bronchoscopy was performed, and she was diagnosed as bronchiolitis obliterans. Owing to the failure of steroid, mycophenolate, and tacrolimus therapy, a skin biopsy was performed and chronic GVHD was diagnosed. Twenty cycles of extracorporeal photopheresis (ECP) was initiated.

On post-transplant month 40, when the patient was 5 years old, increased sweating was noticed; her heart rate was $125 / \mathrm{min}$ and blood pressure was $100 / 65 \mathrm{mmHg}$. There was no palpitation, exophthalmos, tremor, or other symptoms of hyperthyroidism. Her thyroid function tests (Table 2) revealed hyperthyroidism with a free triiodothyronine $\left(\mathrm{fT}_{3}\right)$ level of $8.6 \mathrm{pmol} / \mathrm{L}$ (3.8-6.0 pmol/L). Levels for the following were: free thyroxine $\left(\mathrm{fT}_{4}\right) 17.02 \mathrm{pmol} / \mathrm{L}(7.86-14.41 \mathrm{pmol} / \mathrm{L})$, thyroid-stimulating hormone (TSH) $0.04 \mu \mathrm{lU} / \mathrm{mL} \quad(0.34-5.6$ $\mu \mathrm{lU} / \mathrm{mL})$, thyroglobulin $97.8 \mathrm{ng} / \mathrm{mL}(1.15-50 \mathrm{ng} / \mathrm{mL})$, antithyroid peroxidase antibody (anti-TPO) $36.3 \mathrm{IU} / \mathrm{mL}$ (0-9 IU/ $\mathrm{mL})$, anti-TSH receptor antibody $34.4 \mathrm{IU} / \mathrm{L}(<1 \mathrm{IU} / \mathrm{L})$, and anti-thyroglobulin antibody $<0.9 \mathrm{IU} / \mathrm{mL}(0-4 \mathrm{IU} / \mathrm{mL})$. Spot urine iodide level was $3.8 \mu \mathrm{g} / \mathrm{dL}(10-20 \mu \mathrm{g} / \mathrm{dL})$. Her thyroid ultrasonography (USG) was normal. There was no family history of any thyroid or autoimmune disease. However, her mother (donor) was diagnosed to have Hashimoto's thyroiditis when tested following her daughter's diagnosis. The patient was treated with propranolol and methimazole.

\section{Case 2}

A fifteen-year-old female presented with high-grade fever, diarrhea, recurrent infections, hepatosplenomegaly, and pancytopenia. Her brother had died with a diagnosis of secondary hemophagocytic syndrome due to EpsteinBarr virus infection. Six out of 8 diagnostic criteria for hemaphagocytic syndrome were present in the patient, and the diagnosis was established (7). She was found to be in an accelerated phase of the disease, and the HLH-2004 protocol was initiated. Bone marrow transplantation was performed from the patient's HLA-matched 16-year-old sister. Neutrophil and platelet engraftments were observed on the $11^{\text {th }}$ and $15^{\text {th }}$ days, respectively (Table 1 ).

On post-transplant day 9, the patient developed a maculopapular rash, which was assessed as indicative of acute GVHD. Methylprednisolone was initiated. She responded well and the steroid treatment was tapered and stopped.

Over time, the patient was observed to develop hypertension, neutropenic fever, and grade 3-4 mucositis as HSCT complications.

On the post-transplant month 5, tachycardia was observed (heart rate: $120 / \mathrm{min}$ ), but she had no hypertension (blood pressure: 110/70 mmHg), palpitation, exophthalmos, tremor, or other symptoms of hyperthyroidism. Her thyroid function tests (Table 2) revealed hyperthyroidism with a $\mathrm{fT}_{3}$ level of $11.58 \mathrm{pmol} / \mathrm{L}$ (3.8-6.0 pmol/L), $\mathrm{fT}_{4} 28.98 \mathrm{pmol} / \mathrm{L}$ (12-22 pmol/L), TSH $0.02 \mu \mathrm{lU} / \mathrm{mL}$ (0.34-5.6 $\mu \mathrm{lU} / \mathrm{mL})$, antiTPO antibody $7 \mathrm{IU} / \mathrm{mL}$ (0-9 $\mathrm{IU} / \mathrm{mL})$, anti-TSH receptor antibody $<1 \mathrm{IU} / \mathrm{L}(<1 \mathrm{IU} / \mathrm{L})$, and anti-thyroglobulin antibody $<0.9 \mathrm{IU} / \mathrm{mL}(0-4 \mathrm{IU} / \mathrm{mL})$. Her thyroid USG was normal. She was followed without treatment and had subclinical hypothyroidism on post-transplant $8^{\text {th }}$ month (TSH: 7.92 $\left.\mu \mathrm{lU} / \mathrm{mL}, \mathrm{fT}_{3}: 4.66 \mathrm{pmol} / \mathrm{L}, \mathrm{fT}_{4}: 12.86 \mathrm{pmol} / \mathrm{L}\right)$. Later, on the follow-up at month 22, she had another hyperthyroidism attack with a $\mathrm{fT}_{3} 7.13 \mathrm{pmol} / \mathrm{L}$ (3.8-6.0 pmol/L), $\mathrm{fT}_{4} 23.23$ $\mathrm{pmol} / \mathrm{L}$ (12-22 pmol/L), TSH 0.07 $\mu \mathrm{IU} / \mathrm{mL}$ (0.34-5.6 $\mu \mathrm{lU} / \mathrm{mL})$, and anti-TPO antibody $54.5 \mathrm{lU} / \mathrm{mL}(0-9 \mathrm{lU} / \mathrm{mL})$. She again was followed without treatment for 4 months, at which time her thyroid hormone levels returned to normal. She is now euthyroid at post-transplant month 60 with a $\mathrm{fT}_{3}$ level of $4.59 \mathrm{pmol} / \mathrm{L}$ (3.8-6.0 pmol/L), $\mathrm{fT}_{4} 10.94 \mathrm{pmol} / \mathrm{L}$ (7.86-14.41 $\mathrm{pmol} / \mathrm{L})$, and $\mathrm{TSH} 2.57 \mu \mathrm{lU} / \mathrm{mL}(0.34-5.6 \mu \mathrm{lU} / \mathrm{mL})$.

\section{Case 3}

This male patient had presented with recurrent infections, moniliasis, anemia, and thrombocytopenia to another medical center at age one month and had been hospitalized with a diagnosis of severe combined immunodeficiency disorder (SCID). HSCT was done from his HLA-full matched sister. $\mathrm{T}$ cell engraftment was observed after HSCT; however, thrombocytopenia continued. He was then re-evaluated and diagnosed as Wiskott-Aldrich syndrome. When he was 7 years 
old, a second bone marrow transplantation was done from his sister in our institution. Neutrophil and platelet engraftments were observed on the $13^{\text {th }}$ and $18^{\text {th }}$ days, respectively. He did not have any major HSCT complication (Table 1).

On post-transplant month 7 , he had mild tachycardia (heart rate: 125/min), but he had no hypertension (TA: $90 / 60 \mathrm{mmHg}$ ), palpitation, exophthalmos, tremor, or other symptoms of hyperthyroidism. His thyroid function tests (Table 2) revealed hyperthyroidism with a $\mathrm{fT}_{3}$ level of 11.3 $\mathrm{pmol} / \mathrm{L}$ (3.8-6.0 pmol/L), $\mathrm{fT}_{4} 28.17 \mathrm{pmol} / \mathrm{L}$ (12-22 pmol/L), TSH $0.02 \mu \mathrm{lU} / \mathrm{mL}(0.34-5.6 \mu \mathrm{lU} / \mathrm{mL})$, anti-TPO antibody $38.0 \mathrm{IU} / \mathrm{mL}(0-9 \mathrm{IU} / \mathrm{mL})$, anti-thyroglobulin antibody $<0.9$ $\mathrm{IU} / \mathrm{mL}(0-4 \mathrm{IU} / \mathrm{mL})$, and anti-TSH receptor antibody $<1 \mathrm{IU} / \mathrm{L}$ $(<1 \mathrm{IU} / \mathrm{L})$. His thyroid USG revealed thyroiditis without any nodules. He was treated only with propranolol. He was euthyroid 5 months later (post-transplant month 12) with a fT $6.25 \mathrm{pmol} / \mathrm{L}$ (3.8-6.0 pmol/L), $\mathrm{fT}_{4} 14.59 \mathrm{pmol} / \mathrm{L}$ (7.86$14.41 \mathrm{pmol} / \mathrm{L})$, and TSH $2.41 \mu \mathrm{lU} / \mathrm{mL}(0.34-5.6 \mu \mathrm{lU} / \mathrm{mL})$ without any anti-thyroid treatment.

\section{Case 4}

A sixteen-year-old male presented with left upper extremity pain, anemia, and thrombocytopenia. A bone marrow aspiration was done and he was hospitalized with a diagnosis of acute myeloid leukemia (AML). HSCT was performed at remission from his HLA-full matched sister. Neutrophil and platelet engraftments were observed on the $14^{\text {th }}$ and $17^{\text {th }}$ days, respectively. On post-transplant month 16, there was a mosaic pattern at thorax HRCT. Later on, he developed diarrhea. Steroid and CsA treatment was initiated with a diagnosis of chronic GVHD (Table 1).

\begin{tabular}{|c|c|c|c|c|}
\hline & Case 1 & Case 2 & Case 3 & Case 4 \\
\hline Gender & Female & Female & Male & Male \\
\hline Primary diagnosis & Thalassemia major & Hemophagocytic syndrome & Wiskott-Aldrich syndrome & Acute myeloid leukemia \\
\hline $\begin{array}{l}\text { Age at diagnosis } \\
\text { (primary disease) }\end{array}$ & 10 months & 15 years & 1 month & 16 years \\
\hline Age at HSCT & 29 months & 16 years & 7 years & 16.5 years \\
\hline $\begin{array}{l}\text { Duration between } \\
\text { HSCT and diagnosis of } \\
\text { hyperthyroidism }\end{array}$ & 40 months & 5 months & 7 months & 17 months \\
\hline Donor & HLA-matched mother & HLA-matched sibling & HLA-matched sibling & HLA-matched sibling \\
\hline Stem cell source & Bone marrow & Peripheral blood & Peripheral blood & Peripheral blood \\
\hline CD $34(+)$ cells dose & $3.2 \times 10^{6} / \mathrm{kg}$ & $8.6 \times 10^{6} / \mathrm{kg}$ & $3.7 \times 10^{6} / \mathrm{kg}$ & $4.4 \times 10^{6} / \mathrm{kg}$ \\
\hline Conditioning regimen & $\begin{array}{l}\text { Busulfan i.v. }(16 \mathrm{mg} / \mathrm{kg}) \\
\text { Cyclophosphamide }(200 \mathrm{mg} / \mathrm{kg}) \\
\text { ATG frenius }(20 \mathrm{mg} / \mathrm{kg})\end{array}$ & $\begin{array}{l}\text { Busulfan i.v. }(12.8 \mathrm{mg} / \mathrm{kg}) \\
\text { Cyclophosphamide }(200 \mathrm{mg} / \mathrm{kg}) \\
\text { Etoposide }\left(900 \mathrm{mg} / \mathrm{m}^{2}\right)\end{array}$ & $\begin{array}{l}\text { Busulfan i.v. }(12.8 \mathrm{mg} / \mathrm{kg}) \\
\text { Cyclophosphamide }(200 \\
\mathrm{mg} / \mathrm{kg})\end{array}$ & $\begin{array}{l}\text { Busulfan i.v. (12.8 mg/kg) } \\
\text { Cyclophosphamide (120 } \\
\mathrm{mg} / \mathrm{kg})\end{array}$ \\
\hline GVHD prophylaxis & Cyclosporin A, methotrexate & Cyclosporin A, methotrexate & $\begin{array}{l}\text { Cyclosporin A, } \\
\text { methotrexate }\end{array}$ & $\begin{array}{l}\text { Cyclosporin A, } \\
\text { methotrexate }\end{array}$ \\
\hline $\begin{array}{l}\text { Chimerism analysis } \\
\text { ( }+3^{\text {rd }} \text { month) }\end{array}$ & $98 \%$ & $97 \%$ & $96 \%$ & $96 \%$ \\
\hline Neutrophil engraftment & Day +16 & Day +11 & Day +13 & Day +14 \\
\hline Platelet engraftment & Day +33 & Day +15 & Day +18 & Day +17 \\
\hline $\begin{array}{l}\text { Acute GVHD } \\
\text { ( } \geq \text { grade } 2 \text { ) }\end{array}$ & Yes & No & No & No \\
\hline Chronic GVHD & Severe & No & No & Yes \\
\hline Outcome of HSCT & Living (disease-free) & Living (disease-free) & Living (disease-free) & Living (disease-free) \\
\hline
\end{tabular}


Sağ E et al

Hyperthyroidism After Hematopoietic Stem Cell Transplantation

Table 2. Laboratory tests for hyperthyroidism in the cases and their donors

\begin{tabular}{|c|c|c|c|c|}
\hline Thyroid hormones before HSCT & Case 1 & Case 2 & Case 3 & Case 4 \\
\hline $\mathrm{fT}_{3}(3.8-6.0 \mathrm{pmol} / \mathrm{L})$ & - & $5.87 \mathrm{pmol} / \mathrm{L}$ & $4.46 \mathrm{pmol} / \mathrm{L}$ & $4.35 \mathrm{pmol} / \mathrm{L}$ \\
\hline $\mathrm{fT}_{4}(7.86-14.41 \mathrm{pmol} / \mathrm{L})^{*}(12-22 \mathrm{pmol} / \mathrm{L})^{* *}$ & *11.41 pmol/L & **13.73 pmol/L & **10.87 pmol/L & **16.39 pmol/L \\
\hline TSH $(0.34-5.6 \mu \mathrm{lU} / \mathrm{mL})$ & $3.71 \mu \mathrm{IU} / \mathrm{mL}$ & $2.05 \mu \mathrm{lU} / \mathrm{mL}$ & $2.61 \mu \mathrm{lU} / \mathrm{mL}$ & $2.58 \mu \mathrm{lU} / \mathrm{mL}$ \\
\hline \multicolumn{5}{|l|}{ Thyroid tests at the time of hyperthyroidism } \\
\hline $\mathrm{fT}_{3}(3.8-6.0 \mathrm{pmol} / \mathrm{L})$ & $8.6 \mathrm{pmol} / \mathrm{L}$ & $11.58 \mathrm{pmol} / \mathrm{L}$ & $11.3 \mathrm{pmol} / \mathrm{L}$ & $6.27 \mathrm{pmol} / \mathrm{L}$ \\
\hline $\mathrm{fT}_{4}(7.86-14.41 \mathrm{pmol} / \mathrm{L})^{*}(12-22 \mathrm{pmol} / \mathrm{L})^{* *}$ & *17.02 pmol/L & **28.98 pmol/L & $* * 28.17 \mathrm{pmol} / \mathrm{L}$ & *18.19 pmol/L \\
\hline TSH $(0.34-5.6 \mu \mathrm{lU} / \mathrm{mL})$ & $0.04 \mu \mathrm{lU} / \mathrm{mL}$ & $0.02 \mu \mathrm{lU} / \mathrm{mL}$ & $0.02 \mu \mathrm{lU} / \mathrm{mL}$ & $0.08 \mu \mathrm{lU} / \mathrm{mL}$ \\
\hline Anti-TPO (0-9 IU/mL) & $36.3 \mathrm{IU} / \mathrm{mL}$ & $7 \mathrm{IU} / \mathrm{mL}$ & $38.0 \mathrm{IU} / \mathrm{mL}$ & $94 \mathrm{IU} / \mathrm{mL}$ \\
\hline Anti-thyroglobulin (0-4 IU/mL) & $<0.9 \mathrm{IU} / \mathrm{mL}$ & $<0.9 \mathrm{IU} / \mathrm{mL}$ & $<0.9 \mathrm{IU} / \mathrm{mL}$ & $140.6 \mathrm{IU} / \mathrm{mL}$ \\
\hline TSH receptor antibody $(<1 \mathrm{IU} / \mathrm{L})$ & $34.4 \mathrm{IU} / \mathrm{L}$ & $<1 \mathrm{IU} / \mathrm{L}$ & $<1 \mathrm{IU} / \mathrm{L}$ & $5.9 \mathrm{IU} / \mathrm{L}$ \\
\hline Thyroid USG & Normal & Normal & Thyroiditis & Mild thyroiditis \\
\hline \multicolumn{5}{|l|}{ Thyroid tests of donors } \\
\hline $\mathrm{fT}_{3}(3.8-6.0 \mathrm{pmol} / \mathrm{L})$ & $4.24 \mathrm{pmol} / \mathrm{L}$ & $5.17 \mathrm{pmol} / \mathrm{L}$ & $\begin{array}{l}3.04 \mathrm{pg} / \mathrm{mL} \\
\text { (range: } 2.56-5.01 \mathrm{pg} / \mathrm{mL} \text { ) }\end{array}$ & $4.59 \mathrm{pmol} / \mathrm{L}$ \\
\hline $\mathrm{fT}_{4}(7.86-14.41 \mathrm{pmol} / \mathrm{L})$ & $8.64 \mathrm{pmol} / \mathrm{L}$ & $1.23 \mathrm{~mol} / \mathrm{L}$ & $\begin{array}{l}1.28 \mathrm{ng} / \mathrm{dL} \\
\text { (range: } 0.98-1.30 \mathrm{ng} / \mathrm{dL} \text { ) }\end{array}$ & $12.31 \mathrm{pmol} / \mathrm{L}$ \\
\hline TSH $(0.34-5.6 \mu \mathrm{lU} / \mathrm{mL})$ & $3.08 \mu \mathrm{lU} / \mathrm{mL}$ & $0.83 \mu \mathrm{lU} / \mathrm{mL}$ & $1.60 \mu \mathrm{lU} / \mathrm{mL}$ & $3.0 \mu \mathrm{lU} / \mathrm{mL}$ \\
\hline Anti-TPO (0-9 IU/mL) & $275.7 \mathrm{IU} / \mathrm{mL}$ & $<9 \mathrm{IU} / \mathrm{mL}$ & $<9 \mathrm{IU} / \mathrm{mL}$ & $102.1 \mathrm{IU} / \mathrm{mL}$ \\
\hline Anti-thyroglobulin (0-4 IU/mL) & $<0.9 \mathrm{IU} / \mathrm{mL}$ & $<4 \mathrm{IU} / \mathrm{mL}$ & - & $97.3 \mathrm{IU} / \mathrm{mL}$ \\
\hline TSH receptor autoantibody (<1 IU/L) & $<1 \mathrm{IU} / \mathrm{L}$ & $<1 \mathrm{IU} / \mathrm{L}$ & $<11 \mathrm{U} / \mathrm{L}$ & $<1 \mathrm{IU} / \mathrm{L}$ \\
\hline
\end{tabular}

On the post-transplant month 17 , weight loss was noted. He had no tachycardia (heart rate: 98/min), hypertension (blood pressure: 100/60 $\mathrm{mmHg}$ ), palpitation, tremor, exophthalmos, or other symptoms of hyperthyroidism. His thyroid function tests (Table 2) revealed hyperthyroidism with a $\mathrm{fT}_{3} 6.27 \mathrm{pmol} / \mathrm{L}(3.8-6.0 \mathrm{pmol} / \mathrm{L}), \mathrm{fT}_{4} 18.19 \mathrm{pmol} / \mathrm{L}$ (7.86-14.41 pmol/L), TSH $0.08 \mu \mathrm{lU} / \mathrm{mL} \quad(0.34-5.6 \mu \mathrm{lU} /$ $\mathrm{mL})$, thyroglobulin $140.6 \mathrm{ng} / \mathrm{mL}(1.15-50 \mathrm{ngmL})$, anti-TPO antibody $94 \mathrm{IU} / \mathrm{mL}(0-9 \mathrm{IU} / \mathrm{mL})$, anti-thyroglobulin antibody $<0.9 \mathrm{IU} / \mathrm{mL}(0-4 \mathrm{IU} / \mathrm{mL})$, and anti-TSH receptor antibody $5 \mathrm{IU} / \mathrm{L}(<1 \mathrm{IU} / \mathrm{L})$. His spot urine iodide level was $16.5 \mathrm{\mu g} /$ $\mathrm{dL}(10-20 \mu \mathrm{g} / \mathrm{dL})$. His thyroid USG revealed mild thyroiditis without any nodules. He was followed without treatment. Three weeks later, he was found to have severe weight loss (10 kg in three weeks). Physical examination revealed tachycardia and fine tremors in both hands. His thyroid gland was palpable without any signs of nodules. His thyroid function tests were repeated with a $\mathrm{fT}_{3} 6.71 \mathrm{pmol} / \mathrm{L}$ (3.8$6.0 \mathrm{pmol} / \mathrm{L}), \mathrm{fT}_{4} 46.6 \mathrm{pmol} / \mathrm{L}(7.86-14.41 \mathrm{pmol} / \mathrm{L})$, and $\mathrm{TSH}$ $0.04 \mu \mathrm{lU} / \mathrm{mL}(0.34-5.6 \mu \mathrm{lU} / \mathrm{mL})$. A diagnosis of Hashimoto's thyroiditis was made, and methimazole treatment was initiated. Subsequently, the methimazole dose was tapered gradually and treatment was ceased after 6 months when he became euthyroid.

\section{Discussion}

In our institution, the total number of HSCT in pediatric cases was 313 at the time of this study. Herein, we report four cases of hyperthyroidism after allogeneic HSCT.

Thyroid dysfunction is an important problem in patients treated with HSCT, and several kinds of thyroid disorder such as hypothyroidism, euthyroid sick syndrome, autoimmune thyroiditis, Graves' disease, and even thyroid tumors have been reported. Borgström and Bolme (1) reported a series of 35 allogeneic bone marrow transplant patients; nearly $89 \%$ of them had signs of disturbance in the thyroid axis, mostly due to total body irradiation. In a series of 95 autologous stem-cell transplant recipients from Italy, 15 patients (16\%) had transient subclinical hyperthyroidism and 29 patients had transient low $T_{3}$ syndrome. In another report from the same center evaluating 40 patients, $47.5 \%$ were found to have thyroid dysfunction diagnosed as low $\mathrm{T}_{3}$ syndrome, chronic thyroiditis, transient subclinical hyperthyroidism, 
and subclinical hypothyroidism $(4,5)$. In a different series of 57 stem cell recipients from Japan, 24 had euthyroid sick syndrome (15 of these patients became euthyroid within 8.4 months) and 8 patients had thyrotoxicosis. All of the thyrotoxicosis cases were transient with a mean duration of 2 months; 7 patients with thyrotoxicosis developed hypothyroidism during the follow-up period, with a mean of 12 months after HSCT (2). Au et al (3) reported that 4 out of $222 \mathrm{HSCT}$ patients had autoimmune thyroid disease after treatment and all of these cases had a specific haplotype, HLA-A2-B46-DR9.

In animal models, transfer of HSC can induce remission of some autoimmune conditions. However, HSC can also cause autoimmunity when given to previously healthy mice in experimental studies $(6,8)$. There are previous reports of patients with autoimmune diseases such as systemic lupus erythematosus, Crohn's disease, autoimmune thrombocytopenia, and others who have had complete remission after HSCT which had been done for their associated malignancies (9). On the other hand, there are also cases developing autoimmune diseases such as psoriasis, vitiligo, myasthenia gravis, and autoimmune thyroiditis after receiving HSCT (8).

Hyperthyroidism after allogeneic bone marrow transplantation is rare and is presumably mediated by the transfer of immunocompetent donor lymphocytes to the recipient by HSCT (10). It can occur either by the transfer of pathogenic auto-reactive lymphocyte clone from donor to recipient, or by GVHD (6). There are reports of such cases in the relevant literature $(11,12,13,14)$.

In our patients, we can easily rule out the possibility of radiation injury to the thyroid gland because none of our patients had total body irradiation. In our first case, the donor was an anti-TPO antibody positive, anti TSH receptor antibody negative euthyroid mother. However, the patient had hyperthyroidism with anti-TPO antibody and anti TSH receptor antibody positive, which suggest that the hyperthyroidism did not develop simply because of the transfer of adoptive immunity. The donor was her mother, she was screened after the patient had hyperthyroidism and was found to have a euthyroid Hashimoto's disease. With all these findings and genetic susceptibility, immune dysregulation as a result of GVHD or other factors may have led to expansion of auto-reactive lymphocytes to those which were silent in the donor. Mulligan et al (15) reported a patient with autoimmune hyperthyroidism associated with chronic GVHD after HSCT, but there was no other organ involvement. They speculated that organ-specific GVHD, specific only against thyroid gland, was responsible for the autoimmune hyperthyroidism (15). This same mechanism may have been at work in our second patient, since she was euthyroid before HSCT, had hyperthyroidism without any autoantibody detected after treatment, and her donor was also autoantibody-free but had acute GVHD. Case 3 had a similar clinical and autoantibody status, however, he did not experience either acute or chronic GVHD. Case 4 had hyperthyroidism due to Hashimoto's thyroiditis only 1 month after he was diagnosed to have chronic GVHD, his and his donor's anti-TPO antibody and anti-thyroglobulin antibody were positive.

We conclude that both immune dysregulation and adoptive immunity transfer may have played a role in the development of autoimmune thyroid disease in our patients, but the exact mechanisms need to be elucidated. HSCT can cause thyroid dysfunction more frequently than expected, therefore, the thyroid status of each HSCT patient should be screened before and after the treatment. Further studies are warranted to assess the requirement of screening for thyroid autoantibodies before or after HSCT.

\section{Authorship Contributions}

Informed Consent: It was taken, Concept: Nazlı Gönç, Ayfer Alikaşifoğlu, Design: Erdal Sağ, Nazlı Gönç, Ayfer Alikaşifoğlu, Data Collection or Processing: Erdal Sağ, Nazlı Gönç, Ayfer Alikaşifoğlu, Analysis or Interpretation: Erdal Sağ, Nazlı Gönç, Ayfer Alikaşifoğlu, Alev Özön, Nurgün Kandemir, Barış Kuşkonmaz, Duygu Uçkan, Literature Search: Erdal Sağ, Nazlı Gönç, Ayfer Alikaşifoğlu, Writing: Erdal Sağ, Peer-review: External and Internal peer-reviewed, Financial Disclosure: The authors declared that this study has received no financial support.

\section{References}

1. Borgström B, Bolme P. Thyroid function in children after allogeneic bone marrow transplantation. Bone Marrow Transplant 1994;13:59-64.

2. Kami M, Tanaka Y, Chiba S, Matsumura T, Machida U, Kanda Y, Nakagawa K, Mitsuhashi T, Tanaka Y, Hirai H. Thyroid function after bone marrow transplantation: possible association between immune-mediated thyrotoxicosis and hypothyroidism. Transplantation 2001;71:406-411.

3. Au WY, Hawkins BR, Chan EY, Lie AK, Kung AW, Liang R, Cheng N, Kwong YL. Association of the HLA A2-B46-DR9 haplotype with autoimmune thyroid dysfunction after bone marrow transplantation in Chinese patients. $\mathrm{Br} \mathrm{J}$ Haematol 2001; 115:660-663.

4. Tauchmanova L, Selleri C, De Rosa G, Esposito M, Di Somma C, Orio F, Palomba S, Lombardi G, Rotoli B, Colao A. Endocrine disorders during the first year after autologous stem-cell transplant. Am J Med 2005;118:664-670.

5. Tauchmanova L, Selleri C, Rosa GD, Pagano L, Orio F, Lombardi G, Rotoli B, Colao A. High prevalence of endocrine dysfunction in long-term survivors after allogeneic bone marrow transplantation for hematologic diseases. Cancer 2002;95:1076-1084.

6. Weetman A. Immune reconstitution syndrome and the thyroid. Best Pract Res Clin Endocrinol Metab 2009;23:693702. 
Sağ E et al

Hyperthyroidism After Hematopoietic Stem Cell Transplantation

7. Henter JI, Horne A, Arico M, Egeler RM, Filipovich $A H$, Imashuku S, Ladisch S, McClain K, Webb D, Winiarski J, Janka G. HLH-2004: Diagnostic and therapeutic guidelines for hemophagocytic lymphohistiocytosis. Pediatr Blood Cancer 2007;48:124-131.

8. Sherer $Y$, Shoenfeld $Y$. Autoimmune diseases and autoimmunity post-bone marrow transplantation. Bone Marrow Transplant 1998;22:873-881.

9. Burt RK, Slavin S, Burns WH, Marmont AM. Induction of tolerance in autoimmune diseases by hematopoietic stem cell transplantation: getting closer to a cure? Blood 2002;99:768-784.

10. Tatevossian R, Blair JC, Plowman PN, Savage MO, Shankar AG. Thyrotoxicosis after matched unrelated bone marrow transplantation. J Pediatr Hematol Oncol 2004;26:529-531.

11. Aldouri MA, Ruggier R, Epstein O, Prentice HG. Adoptive transfer of hyperthyroidism and autoimmune thyroiditis following allogeneic bone marrow transplantation for chronic myeloid leukaemia. Br J Haematol 1990;74:118119.

12. Yamamori I, Kanie $T$, Maeda $N$, Kodera $Y$, Matsuyama $\mathrm{T}$, Hasegawa $\mathrm{H}$. Appearance of thyroid stimulating and blocking immunoglobulins after bone marrow transplantation: presentation of two contrasting cases. Endocr J 2004;51:439-443.

13. Berisso GA, van Lint MT, Bacigalupo A, Marmont AM. Adoptive autoimmune hyperthyroidism following allogeneic stem cell transplantation from an HLA-identical sibling with Graves' disease. Bone Marrow Transplant 1999;23:1091-1092.

14. Takeshita A, Shinjo K, Ohno R. Graves disease after bone marrow transplantation. Ann Intern Med 1999;131:157.

15. Mulligan SP, Joshua DE, Joasoo A, Kronenberg $H$. Autoimmune hyperthyroidism associated with chronic graftversus-host disease. Transplantation 1987;44:463-464. 